\title{
Immunohistochemical Investigation of HER/AKT/mTOR Pathway and Cellular Adhesion Molecules in Urothelial Carcinomas
}

\author{
Nikolaos Koletsas, ${ }^{1}$ Triantafyllia Koletsa, ${ }^{2}$ Spyros Choidas, ${ }^{1}$ \\ Konstantinos Anagnostopoulos, ${ }^{3}$ Stavros Touloupidis, ${ }^{4}$ Thomas Zaramboukas, ${ }^{2}$ \\ Georgia Raptou, ${ }^{2}$ Nikolaos Papadopoulos, ${ }^{5}$ and Maria Lambropoulou ${ }^{5}$ \\ ${ }^{1}$ Urology Department, Interbalkan Medical Center, Thessaloniki, Greece \\ ${ }^{2}$ Pathology Department, Medical School, Aristotle University of Thessaloniki, Thessaloniki, Greece \\ ${ }^{3}$ Laboratory of Biochemistry, School of Medicine, Democritus University of Thrace, Alexandroupolis, Greece \\ ${ }^{4}$ Urology Department, School of Medicine, Democritus University of Thrace, Alexandroupolis, Greece \\ ${ }^{5}$ Laboratory of Histology-Embryology, School of Medicine, Democritus University of Thrace, Alexandroupolis, Greece
}

Correspondence should be addressed to Triantafyllia Koletsa; koletsa@med.auth.gr

Received 30 October 2016; Accepted 29 December 2016; Published 22 January 2017

Academic Editor: Marco Volante

Copyright (C) 2017 Nikolaos Koletsas et al. This is an open access article distributed under the Creative Commons Attribution License, which permits unrestricted use, distribution, and reproduction in any medium, provided the original work is properly cited.

\begin{abstract}
Background. Several investigators have suggested the possibility that the expression of both EGFR and HER2 could be utilized for molecularly targeted therapy in urinary bladder cancer. We tried to evaluate the expression of HER2 and EGFR and activation of the AKT/PTEN/mTOR pathway in urothelial carcinomas and if there is any association between them and cellular adhesion molecules (CAMs). Materials and Methods. Forty-one paraffin-embedded urothelial cancer tissue blocks were collected. Immunostains for HER2, EGFR, MIB1, phospho-AKT, PTEN, phospho-mTOR, e-cadherin, p-cadherin, and b-catenin were performed on tissue microarrays sections. The immunohistochemical results were correlated with clinicopathological parameters. Results. The overexpression of HER2 was found in $19.6 \%$ of the cases and it was associated with high grade tumors with a high mitotic index and phosphorylation of AKT and mTOR. Muscle-invasive tumors presented both cytoplasmic and nuclear losses of PTEN expression. There was no association between HER/AKT/mTOR pathway activation and CAM expression. Although cadherins were often coexpressed, only p-cadherin immunoreactivity was associated with tumor grade and high proliferative index. Conclusions. HER2 overexpression is found in a respective proportion of urothelial carcinomas. $\mathrm{P}$-cadherin expression is associated with high grade UCs but it is not affected by HER2 overexpression or by activation of HER/AKT/mTOR pathway.
\end{abstract}

\section{Background}

The human epidermal growth factor receptors (HER) protein family consists of four different transmembrane receptors (HER1-HER4). HER1/EGFR and HER2/c-erb-B2 are the most thoroughly investigated family members and have been documented to be involved in the pathogenesis of several types of cancers. In urothelial carcinomas HER1 and HER2 expression has been implicated in tumor aggressiveness, poor outcome, or even pathogenesis [1-4]. In recent years, their importance has been emphasized due to the development of targeted anti-HER therapy.
The dimerization of HER members leads to the activation of intracellular RAS/MEK/ERK [5] and PI3K/AKT/PTEN/ mTOR $[6,7]$ pathways which plays an important role in cell proliferation, angiogenesis, invasion, and metastasis. The $\mathrm{PI} 3 \mathrm{~K} / \mathrm{AKT} / \mathrm{PTEN} / \mathrm{mTOR}$ pathway is considered to be essential for cell growth, survival, cell motility, and angiogenesis [8-11]. The activation of this pathway has been implicated in carcinogenesis or malignant potential of several cancers, including urothelial ones $[12,13]$.

The categorization of urothelial carcinomas is based on grading and muscle invasion. The majority of urothelial 
carcinomas are noninvasive tumors of low grade [14]. Muscle invasion carcinomas are characterized by mutations in TP53, RB1, and PIK3CA genes and deletions in PTEN gene $[4,15]$ and loss of e-cadherin [16]. Cell adhesion molecules (CAMs) are required for maintaining a normal epithelial phenotype and abnormalities in their expression have been related to cancer progression [16].

The present study was conducted to investigate the expression of EGFR and HER2 proteins, as well as intracellular signaling molecules in sections of urothelial carcinomas by immunohistochemistry, to analyze e-cadherin, $\mathrm{p}$ cadherin, and b-catenin expression in low and high grade urothelial carcinomas, and to correlate the immunohistochemical results with clinicopathologic parameters.

\section{Materials and Methods}

A total of 41 archived cases of urothelial bladder carcinomas were included in this study. Clinical data and complete follow-up were known in 23 patients. The pertinent hematoxylin and eosin (HE) stained sections were retrieved and reevaluated by pathologist and the representative neoplastic areas corresponding to tumor classification and grading were marked for tissue microarrays formation.

2.1. Construction of Tissue Microarrays (TMAs). Formalinfixed paraffin-embedded (FFPE) tissue samples from urothelial tumors (paraffin blocks) were collected retrospectively. TMA blocks were constructed with the Alphelys Minicore 3 Tissue Microarray system (Plaisir, France). Each tumor was represented by 3 tissue cores, $1 \mathrm{~mm}$ in diameter, which were obtained from the marked representative areas of neoplasms and reembedded in recipient paraffin blocks. TMAs also contained cores from placenta, tonsil and normal thyroid, breast, and renal and colon tissue, used as control markers and for section orientation. Four-micrometer-thick sections were obtained and stained by immunohistochemical (IHC) method.

2.2. Immunohistochemistry. IHC staining was performed on freshly cut sections. Primary antibodies against HER2 (polyclonal, Dako, Glostrup, Denmark), EGFR (clone 31G7, Invitrogen, Carlsbad, CA, USA), phospho-AKT 1/2/3 (Thr308)R (polyclonal, Santa Cruz Biotechnology, Santa Cruz, CA, USA), PTEN (clone 6h2.1, Dako, Denmark), phospho-mTOR (Ser2448) (clone 49F9, Cell Signaling Technology, Danvers, MA, USA), Ki67 (clone MIB1, Dako, Denmark), E-cadherin (\#610181, BD Transduction Laboratories, San Jose, CA, USA), and beta-catenin (\#610153, BD Transduction Laboratories, San Jose, CA, USA) were used. IHC stains were performed on a Bond automated stainer (Dako).

2.3. Immunohistochemical Evaluation. There is no standard protocol or guidelines for the estimation of HER2 expression in urothelial carcinomas or what the most appropriate cutoff value is. The HER2 immunostain scoring was performed based on the guidelines of the American Society of Clinical Oncology/College of American Pathologist (0: no staining, 1+: incomplete membranous staining, $2+$ : complete but weak or moderate membranous staining in $>10 \%$ of cells, and $3+$ :
TABLE 1: Clinicopathologic characteristics of the tumors.

\begin{tabular}{lcc}
\hline & $N$ & $\%$ \\
\hline pTa & 22 & 53.6 \\
pT1 & 6 & 14.7 \\
pT2 & 9 & 21.9 \\
pT3 & 2 & 4.9 \\
pT4 & 2 & 4.9 \\
Low grade & 19 & 46.3 \\
High grade & 22 & 53.7 \\
$<3$ cm & 24 & 58.5 \\
$\geq 3 \mathrm{~cm}$ & 17 & 41.5 \\
One lesion & 20 & 48.8 \\
Multifocal & 17 & 41.4 \\
Missing data & 4 & 9.8 \\
\hline
\end{tabular}

strong membranous staining in more than $10 \%$ of the cells) [17]. There is no standard protocol for EGFR evaluation. EGFR expression was considered as positive when complete membrane positivity was observed in a percentage of $>10 \%$ of the cells, as it was used before [18]. For phospho-AKT (pAKT) and PTEN both percentage of positive cells and intensity for nuclear and cytoplasmic immunoreaction were evaluated. The percentage of positive tumor cells (0-100\%) was multiplied by dominant staining intensity (1: weak, 2 : medium, and 3: intense) and a cutoff value based on the median tumor $H$-score was used, as described by GonzalezRoibon et al. [19]. phospho-mTOR (pmTOR) immunostaining was considered negative when expression was observed in $<10 \%$ of cells and positive if immunoreactivity was found in $\geq 10 \%$ of cells [20]. A tumor was considered to have a high mitotic index when there were positive cells to Ki67/MIB1 antibody in a percentage of $>20 \%$.

In 35 cases with adequate specimen immunohistochemistry for CAM expression was also applied. Eighteen out of 35 tumors were of high grade while only ten were invasive. Tumors with positive cells in a percentage $>10 \%$ was considered positive for e-cadherin, $\mathrm{p}$-cadherin, and bcatenin. According to the intensity of staining tumors were categorized as weak, moderate, and strong [21].

2.4. Statistical Analysis. The statistical software package SPSS v. 21 was used for statistical analyses. Chi-square test was employed to test the dependence between different parameters. Values of 0.05 or less were considered to be statistically significant.

\section{Results}

3.1. Clinicopathological Characteristics of Patients. Forty-one patients were included in the study, 32 males and 9 females. Their mean age was 68 years (range 47-87). Tumor characteristics are presented in Table 1 . Nineteen of the carcinomas were low grade $(46.3 \%)$ while $22(53.7 \%)$ were of high grade. All tumors with advanced stage were of high grade. Almost one-third of the cases were muscle-invasive tumors. Patients with stages pTa and pT1 were treated either with epirubicin or BCG depending on histological grade, tumor size, 
and multiplicity. Radical cystectomy was followed for those patients with invasive tumors. Chemotherapy was added in two cases with metastatic disease. A third patient underwent only radiotherapy due to the small size of a solitary lesion located on the frontal bladder wall.

3.2. Immunohistochemical Distribution of the Markers. The immunohistochemical results are summarized in Table 2. HER2 overexpression (3+) was found in 8 cases (19.6\%), seven of which were of high grade $(p=0.032)$. HER2 $2+$ and $3+$ immunoscores accounted for almost $46.4 \%$ and were mainly found in high grade tumors $(p=0.179)$. Sixty percent of HER2 $3+$ tumors measured more than $3 \mathrm{~cm}(p=0.039)$. HER2 expression exhibited positive correlation with pAKT cytoplasmic and nuclear immunoreactivity $(p=0.049$ and $p=0.021$, resp.) (Figure 1). Moreover, in 12 out of 19 HER2 positive cases, pmTOR was coexpressed $(p=0.021)$. The majority of the HER2 positive cases had high mitotic index $(p=0.021)$, defined as $>20 \%$ positive cells to Ki67/MIB1 antibody.

HER2/EGFR coexpression was observed in four cases (9.75\%). There was no association between EGFR expression (14/41, 34.14\%) or HER2/EGFR coexpression (4/41, 9.75\%) and the examined clinicopathologic parameters $\left(x^{2}, p>\right.$ $0.05)$.

Fifteen out of eighteen high grade cases (83.3\%) presented high mitotic indices $(p=0.001)$. MIB1 positivity was associated with HER2 positivity $(p=0.021)$ and pmTOR cytoplasmic $(p=0.035)$ expression $(p<0.001)$.

Loss of PTEN cytoplasmic expression was found mainly in muscle-invasive tumors $(p=0.001$ ) (Table 3). A loss of PTEN expression was defined as simultaneous lack of nuclear and cytoplasmic immunoreactivity. Muscle-invasive tumors presented commonly a loss of PTEN expression ( $p=$ $0.023)$. None of the cases without cytoplasmic PTEN staining exhibited cytoplasmic expression of pAKT $(p=0.032)$. PTEN cytoplasmic expression was positively associated with the cytoplasmic expression of pmTOR protein $(p=0.01)$. However, lack of PTEN nuclear immunoreactivity was not associated with any of the other studied markers, apart from a trend of negative association observed with pAKT nuclear expression $(p=0.09)$. In three cases PTEN was immunoreactive in membranes, as well.

The majority of the muscle-invasive tumors (pT2-pT4) $(9 / 13,69.2 \%)$ expressed pmTOR protein compared to pTapT1 urothelial carcinomas $(p=0.045)$ (Table 3). Cytoplasmic pmTOR expression was associated with high MIB1 labeling index $(p=0.035)$ and neoplastic invasion $(p=0.045)$. Notably, membranous immunoreactivity to pmTOR was found in seven cases.

In this cohort, HER2 overexpression along with pAKT nuclear expression, both nuclear and cytoplasmic PTEN deletion and pmTOR expression, was found in three of the patients. Two of them coexpressed the EGFR protein, and they had the worst prognosis.

Expression of e-cadherin and p-cadherin was observed in $54.3 \%(19 / 35)$ and $41.2 \%(14 / 34)$ of the cases, respectively (Table 2). There was no association between CAM expression and tumor size (Table 3 ) or aggressive behavior $\left(x^{2}, p>0.05\right)$.
TABLE 2: Immunohistochemical distribution of the examined markers.

\begin{tabular}{|c|c|c|}
\hline & $N$ & $\%$ \\
\hline \multicolumn{3}{|l|}{ HER2 } \\
\hline 0 & 8 & 19.5 \\
\hline $1+$ & 14 & 34.1 \\
\hline $2+$ & 11 & 26.8 \\
\hline $3+$ & 8 & 19.6 \\
\hline \multicolumn{3}{|l|}{ EGFR } \\
\hline Negative & 27 & 65.9 \\
\hline Positive & 14 & 34.1 \\
\hline \multicolumn{3}{|l|}{ pAKT nuclear } \\
\hline Negative & 17 & 41.5 \\
\hline Positive & 24 & 58.5 \\
\hline \multicolumn{3}{|c|}{ pAKT cytoplasmic } \\
\hline Negative & 29 & 70.7 \\
\hline Positive & 12 & 29.3 \\
\hline \multicolumn{3}{|c|}{ PTEN cytoplasmic } \\
\hline Negative & 19 & 47.5 \\
\hline Positive & 21 & 52.5 \\
\hline Missing data & 1 & - \\
\hline \multicolumn{3}{|l|}{ PTEN nuclear } \\
\hline Negative & 31 & 77.5 \\
\hline Positive & 9 & 22.5 \\
\hline Missing data & 1 & - \\
\hline \multicolumn{3}{|c|}{ PTEN nuclear/cytoplasmic } \\
\hline Negative & 10 & 25 \\
\hline Positive & 30 & 75 \\
\hline Missing data & 1 & - \\
\hline \multicolumn{3}{|l|}{ pmTOR } \\
\hline Negative & 19 & 46.3 \\
\hline Positive & 22 & 53.7 \\
\hline \multicolumn{3}{|l|}{ MIB1 } \\
\hline$\leq 20$ & 23 & 56.1 \\
\hline$>20$ & 18 & 43.9 \\
\hline \multicolumn{3}{|l|}{ e-cadherin } \\
\hline Negative & 16 & 45.7 \\
\hline Positive & 19 & 54.3 \\
\hline Missing data & 6 & - \\
\hline \multicolumn{3}{|l|}{ p-cadherin } \\
\hline Negative & 20 & 58.8 \\
\hline Positive & 14 & 41.2 \\
\hline Missing data & 7 & - \\
\hline \multicolumn{3}{|l|}{ b-catenin } \\
\hline Negative & 10 & 28.6 \\
\hline Positive & 25 & 71.4 \\
\hline Missing data & 6 & - \\
\hline
\end{tabular}

Fifteen out of 19 tumors with stage pTa were negative to pcadherin antibody, reflecting a trend of association between stage and protein expression of this marker $\left(x^{2}, p=0.07\right)$. A positive association was observed between e-cadherin and p-cadherin expression ( $p=0.002$ ) (Figure 2 ). The majority 


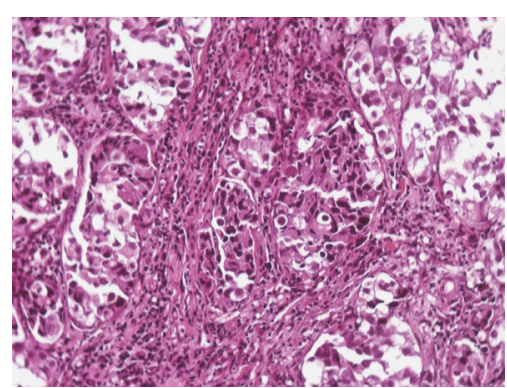

(a)

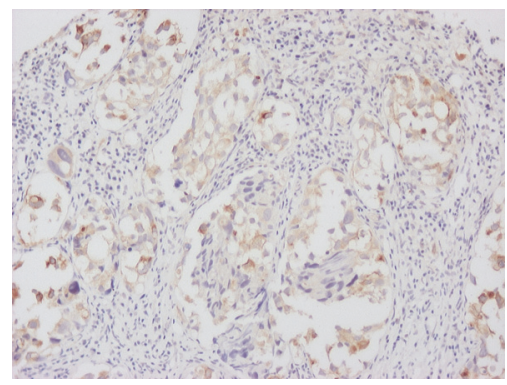

(d)

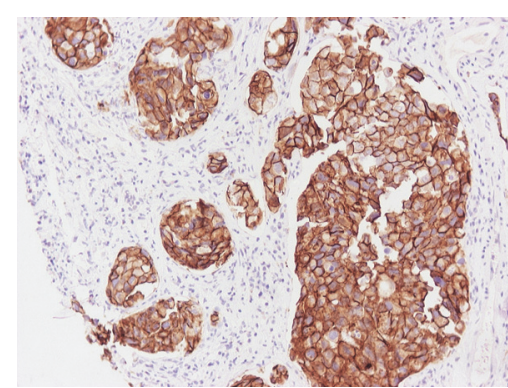

(b)

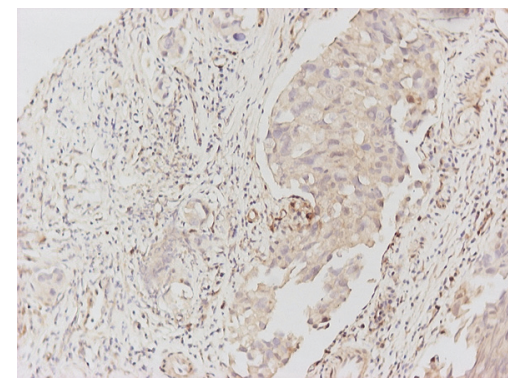

(e)

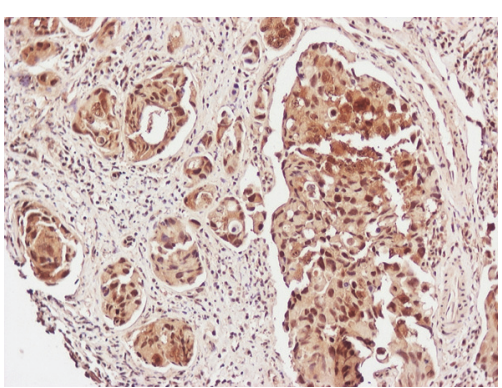

(c)

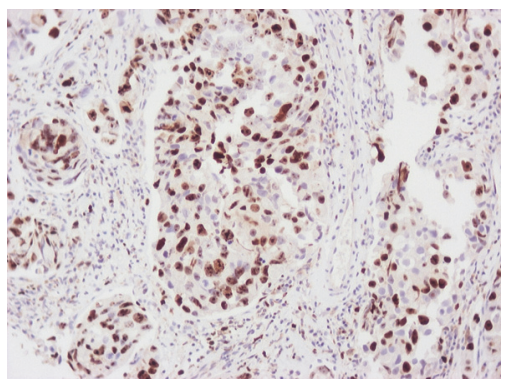

(f)

FIGURE 1: A case of high grade infiltrative urothelial carcinoma (a) presenting HER2 overexpression (b), pAKT (c) and pmTOR (d) expression, loss of PTEN expression (e), and high MIB1 labeling index (f). ((a) HE ×200; (b-f) IHC ×200).

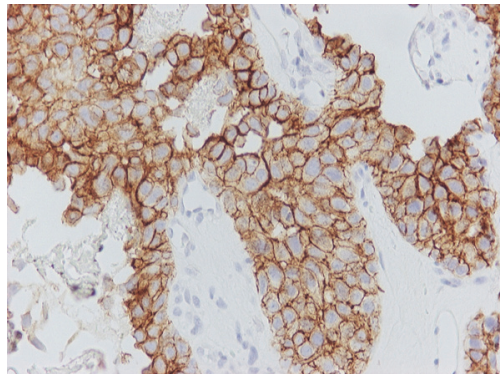

(a)

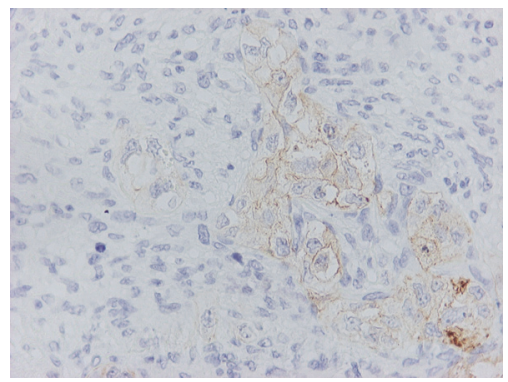

(d)

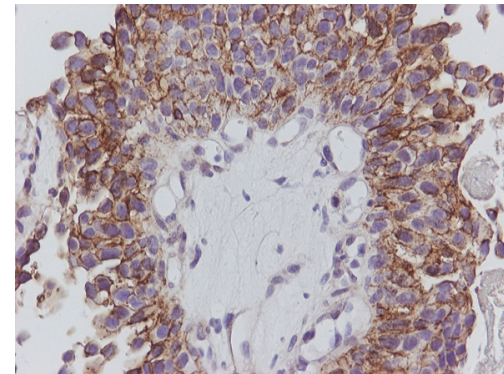

(b)

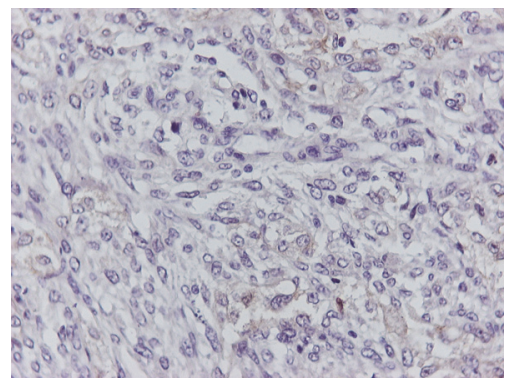

(e)

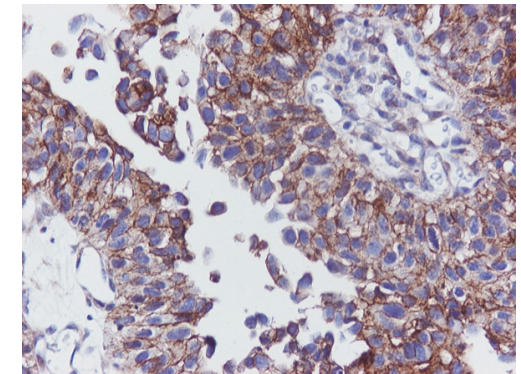

(c)

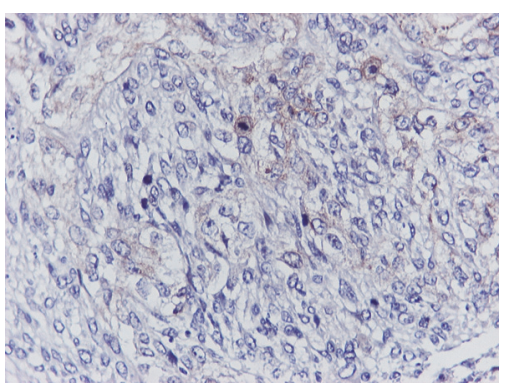

(f)

FIGURE 2: CAM expression in different urothelial tumors. A case of noninfiltrative high grade urothelial carcinoma positive for e-cadherin (a), p-cadherin (b), and b-catenin (c) markers, in contrast to high grade infiltrative urothelial carcinoma with sarcomatoid features, exhibited no or weak expression in few cells to e-cadherin (d), p-cadherin (e), and b-catenin (f) antibodies ((a-f) IHC $\times 400)$.

of the cases $(25 / 35,71.4 \%)$ expressed b-catenin. E-cadherin and $\mathrm{p}$-cadherin positive tumors were mainly of high grade $\left(x^{2}, p=0.037\right.$ and $p=0.002$, resp.). P-cadherin expression was mostly found in tumors with high mitotic indices $(\mathrm{MIB1}>20 \%)\left(x^{2}, p=0.007\right)$. There was no association between CAM expression and muscle-invasive tumors $\left(x^{2}\right.$, $p>0.05)$, pointing out the small sample of the tumors examined for these adhesion molecules. Of note, a case of sarcomatoid carcinoma included in the study exhibited no immunoreactivity to antibodies for CAMs (Figure 2). 
TABLE 3: Immunoexpression of the markers according to tumors invasiveness.

\begin{tabular}{|c|c|c|c|}
\hline & $\begin{array}{l}\text { Muscle-invasive } \\
\text { UC (pT2-pT4) }\end{array}$ & $\begin{array}{c}\text { Non-muscle-invasive } \\
\text { UC (pTa-pT1) }\end{array}$ & $\begin{array}{c}p \\
\text { value }\end{array}$ \\
\hline \multicolumn{4}{|l|}{ HER2 } \\
\hline 0 & 4 & 4 & \multirow{4}{*}{0.252} \\
\hline 1 & 3 & 11 & \\
\hline 2 & 2 & 9 & \\
\hline 3 & 4 & 4 & \\
\hline \multicolumn{4}{|l|}{$\overline{\text { EGFR }}$} \\
\hline Negative & 10 & 17 & \multirow{2}{*}{0.308} \\
\hline Positive & 3 & 11 & \\
\hline \multicolumn{4}{|l|}{ pAKT } \\
\hline Nuclear positive & 16 & 8 & \multirow{2}{*}{0.790} \\
\hline Nuclear negative & 5 & 12 & \\
\hline \multicolumn{4}{|l|}{ pAKT } \\
\hline Cytopl positive & 2 & 9 & \multirow{2}{*}{0.315} \\
\hline Cytopl negative & 11 & 19 & \\
\hline \multicolumn{4}{|l|}{ pmTOR } \\
\hline Negative & 9 & 10 & \multirow{2}{*}{0.045} \\
\hline Positive & 4 & 18 & \\
\hline \multicolumn{4}{|l|}{ PTEN } \\
\hline Nuclear positive & 1 & 8 & \multirow{3}{*}{0.120} \\
\hline Nuclear negative & 12 & 19 & \\
\hline Missing data & 0 & 1 & \\
\hline \multicolumn{4}{|l|}{ PTEN } \\
\hline Cytopl positive & 3 & 18 & \multirow{3}{*}{0.01} \\
\hline Cytopl negative & 10 & 9 & \\
\hline Missing data & 0 & 1 & \\
\hline \multicolumn{4}{|l|}{ e-cadherin } \\
\hline Negative & 4 & 12 & \multirow{3}{*}{0.668} \\
\hline Positive & 6 & 13 & \\
\hline Missing data & 3 & 3 & \\
\hline \multicolumn{4}{|l|}{ p-cadherin } \\
\hline Negative & 4 & 16 & \multirow{3}{*}{0.307} \\
\hline Positive & 5 & 9 & \\
\hline Missing data & 4 & 3 & \\
\hline \multicolumn{4}{|l|}{ b-catenin } \\
\hline Negative & 2 & 8 & \multirow{3}{*}{0.518} \\
\hline Positive & 8 & 17 & \\
\hline Missing data & 3 & 3 & \\
\hline
\end{tabular}

Cytopl: cytoplasmic; UC: urothelial carcinomas.

\section{Discussion}

Over the last decade, two of the HER family members, HER1/EGFR and HER2, have been researched extensively in the context of various types of cancer. Apart from their role in tumor proliferation, infiltration, and metastatic potential [22], the increasing interest in them derives from being targets of newly developed and FDA approved therapies.
HER2 expression in urothelial carcinomas has been reported in several percentages ranging from $9 \%$ to $74.8 \%$ [23-25]. This discrepancy is mainly attributed to the differences in the used cutoffs and the constitution of cohorts, that is, the aggressiveness of the cases included in a study.

Notably, many studies defined HER2 overexpression as both HER2 $2+$ and 3+ immunoscores in urothelial carcinomas [25], as opposed to breast carcinomas. Scoring of the HercepTest corresponds to the number of extracellular domains located in the membrane [26]. In several types of cancer, such as breast carcinomas or gastric/gastroesophageal carcinomas, there are guidelines for protein expression evaluation and criteria for determining overexpression [17, 27, 28]. In urothelial carcinomas there are varying methods and cutoffs used by several studies. However, in a large cohort, Laé et al. [25] found that a true HER2 overexpression in bladder carcinomas corresponded to HER2 gene amplification, being defined in the same way as in breast cancer.

In the present study, HER2 $3+$ was observed in $19.6 \%$ of the cases, while HER2 $2+$ and $3+$ account for $46.4 \%$. The observed association between tumor grade and HER2 expression has been previously well documented [29, 30]. In addition, the aforementioned associations between HER2 expression and tumor size, pAKT, and pmTOR expression indicate that the PI3K/AKT/mTOR pathway could be activated by HER dimerization. Indeed, cases with HER 2 overexpression (HER2 3+) were of high grade and they were usually characterized by $\mathrm{pAKT}$ and pmTOR protein overexpression and PTEN nuclear deletion. This was the immunophenotypic profile of the tumors of the two patients that presented the worst prognosis in this study. In one of these tumors, EGFR coexpression was also observed, which suggests that both AKT/PTEN/TOR and RAS/MEK/ERK pathways were activated. The increased interest to personalize therapy leads to a thorough investigation of patients who will benefit the most from a particular treatment. Hence, these patients could be the most appropriate candidates for targeted therapy, when conventional therapy fails, taking into account the side effects of these therapeutic options [31].

PTEN deletion affected more often the nucleus than the cytoplasm, a finding in line with those of previous reported studies [32, 33], and it could be found in noninfiltrating tumors but it occurs more often in muscle-invasive ones [34-36]. PTEN deletion is also observed in tumors without HER/AKT/mTOR pathway activation $[35,37]$, as found in our study, suggesting that PTEN loss is not always responsible for AKT activation, adding that it may be involved in other pathways [37], as it is known that different intracellular pathways are linked through cross-talking [38] or that synergistic action of different pathways is essential for carcinogenesis or aggressive biological behavior [39].

It has been reported that inactivation of p53 and inactivation of PTEN are the principal adverse prognostic markers [40]. In addition, PTEN deletion in combination with altered p53 leads to deregulation of the mTOR pathway and, consequently, reinforces the use of newly therapeutic agents, such as rapamycin [41], everolimus (RAD001) [42], or a combination of mTOR and PI3K inhibitors [43-45]. 
The cadherins are a group of membrane glycoprotein and the mediators of cell to cell adhesion. E-cadherin, which is an epithelial-specific cadherin, plays a major role in the selective adhesion of cells in epithelial tissue and is necessary for the maintenance of normal epithelial cells integrity. Abnormal expression of $\mathrm{p}$-cadherin has been associated with an invasive and aggressive phenotype of UCs and it has been hypothesized that it may act as a key effector of muscle invasion [46]. CAMs in this study was generally coexpressed, especially in high grade tumors, but they were not associated with infiltration status, a finding that may be biased due to small sample of infiltrative tumors. As previously mentioned [46], and confirmed by this study, p-cadherin seems to be commonly expressed in high grade tumors exhibiting high mitotic index. HER pathway does not appear to affect CAM expression.

In conclusion, HER 2 overexpression is found in a respective proportion of urothelial carcinomas and it seems to characterize an aggressive tumor behavior. The combination of pAKT and pmTOR expression along with a loss of PTEN expression is associated with adverse clinicopathological characteristics. P-cadherin is associated with high grade UCs but its expression is not affected by HER2 overexpression or by activation of HER/AKT/mTOR pathway.

\section{Competing Interests}

The authors declare that they have no competing interests.

\section{References}

[1] C. Bolenz, S. F. Shariat, P. I. Karakiewicz et al., "Human epidermal growth factor receptor 2 expression status provides independent prognostic information in patients with urothelial carcinoma of the urinary bladder," BJU International, vol. 106, no. 8, pp. 1216-1222, 2010.

[2] N.-H. Chow, H.-S. Liu, E. I. C. Lee et al., "Significance of urinary epidermal growth factor and its receptor expression in human bladder cancer," Anticancer Research, vol. 17, no. 2B, pp. 12931296, 1997.

[3] M. Enache, C. E. Simionescu, and A. Stepan, "EGFR and Her2/neu immunoexpression in papillary urothelial bladder carcinomas," Romanian Journal of Morphology and Embryology, vol. 54, no. 1, pp. 137-141, 2013.

[4] P. Korkolopoulou, G. Levidou, E.-A. Trigka et al., "A comprehensive immunohistochemical and molecular approach to the $\mathrm{PI} 3 \mathrm{~K} / \mathrm{AKT} / \mathrm{mTOR}$ (phosphoinositide 3-kinase/v-akt murine thymoma viral oncogene/mammalian target of rapamycin) pathway in bladder urothelial carcinoma," BJU International, vol. 110, no. 11, pp. E1237-E1348, 2012.

[5] M. Campiglio, S. Ali, P. G. Knyazev, and A. Ullrich, "Characteristics of EGFR family-mediated HRG signals in human ovarian cancer," Journal of Cellular Biochemistry, vol. 73, no. 4, pp. 522$532,1999$.

[6] N. V. Sergina, M. Rausch, D. Wang et al., "Escape from HERfamily tyrosine kinase inhibitor therapy by the kinase-inactive HER3," Nature, vol. 445, no. 7126, pp. 437-441, 2007.

[7] S. P. Soltoff, K. L. Carraway III, S. A. Prigent, W. G. Gullick, and L. C. Cantley, "ErbB3 is involved in activation of phosphatidylinositol 3-kinase by epidermal growth factor," Molecular and Cellular Biology, vol. 14, no. 6, pp. 3550-3558, 1994.
[8] R. J. Shaw and L. C. Cantley, "Ras, PI(3)K and mTOR signalling controls tumour cell growth," Nature, vol. 441, no. 7092, pp. 424-430, 2006.

[9] K. D. Courtney, R. B. Corcoran, and J. A. Engelman, “The PI3K pathway as drug target in human cancer," Journal of Clinical Oncology, vol. 28, no. 6, pp. 1075-1083, 2010.

[10] C. Bartholomeusz and A. M. Gonzalez-Angulo, "Targeting the PI3K signaling pathway in cancer therapy," Expert Opinion on Therapeutic Targets, vol. 16, no. 1, pp. 121-130, 2012.

[11] H. Pópulo, J. M. Lopes, and P. Soares, “The mTOR signalling pathway in human cancer," International Journal of Molecular Sciences, vol. 13, no. 2, pp. 1886-1918, 2012.

[12] M. A. Knowles, F. M. Platt, R. L. Ross, and C. D. Hurst, "Phosphatidylinositol 3-kinase (PI3K) pathway activation in bladder cancer," Cancer and Metastasis Reviews, vol. 28, no. 3-4, pp. 305-316, 2009.

[13] J. M. Askham, F. Platt, P. A. Chambers, H. Snowden, C. F. Taylor, and M. A. Knowles, "AKT1 mutations in bladder cancer: identification of a novel oncogenic mutation that can co-operate with E17K," Oncogene, vol. 29, no. 1, pp. 150-155, 2010.

[14] M. Burger, W. Oosterlinck, B. Konety et al., "ICUD-EAU international consultation on bladder cancer 2012: non-muscleinvasive urothelial carcinoma of the bladder," European Urology, vol. 63, no. 1, pp. 36-44, 2013.

[15] S. Z. Millis, D. Bryant, G. Basu et al., "Molecular profiling of infiltrating urothelial carcinoma of bladder and nonbladder origin," Clinical Genitourinary Cancer, vol. 13, no. 1, pp. e37-e49, 2015.

[16] W. Sun and G. A. Herrera, "E-cadherin expression in invasive urothelial carcinoma," Annals of Diagnostic Pathology, vol. 8, no. 1, pp. 17-22, 2004.

[17] A. C. Wolff, M. E. Hammond, D. G. Hicks et al., "American Society of Clinical Oncology; College of American Pathologists. Recommendations for human epidermal growth factor receptor 2 testing in breast cancer: American Society of Clinical Oncology/College of American Pathologists clinical practice guideline update," Journal of Clinical Oncology, vol. 31, no. 31, pp. 3997-4013, 2013.

[18] S. Leibl, R. Zigeuner, G. Hutterer, T. Chromecki, P. Rehak, and C. Langner, "EGFR expression in urothelial carcinoma of the upper urinary tract is associated with disease progression and metaplastic morphology," Acta Pathologica, Microbiologica, et Immunologica Scandinavica, vol. 116, no. 1, pp. 27-32, 2008.

[19] N. D. Gonzalez-Roibon, A. Chaux, T. Al-Hussain et al., "Dysregulation of mammalian target of rapamycin pathway in plasmacytoid variant of urothelial carcinoma of the urinary bladder," Human Pathology, vol. 44, no. 4, pp. 612-622, 2013.

[20] J. Afonso, A. Longatto-Filho, V. M. Da Silva, T. Amaro, and L. L. Santos, "Phospho-mTOR in non-tumour and tumour bladder urothelium: pattern of expression and impact on urothelial bladder cancer patients," Oncology Letters, vol. 8, no. 4, pp. 14471454, 2014

[21] S. T. dos Reis, K. R. M. Leite, A. M. Neto et al., "Immune expression of E-cadherin and $\alpha, \beta$ and $\gamma$-catenin adhesion molecules and prognosis for upper urinary tract urothelial carcinomas," International Braz J Urol, vol. 38, no. 4, pp. 466473, 2012.

[22] S. Kaptain, L. K. Tan, and B. Chen, "Her-2/neu and breast cancer," Diagnostic Molecular Pathology, vol. 10, no. 3, pp. 139152, 2001.

[23] D. A. Pigott, A. J. Proctor, M. E. Eydmann et al., "Amplification and over-expression of c-erbB-2 in transitional cell carcinoma 
of the urinary bladder," British Journal of Cancer, vol. 63, no. 4, pp. 601-608, 1991.

[24] C. Wülfing, D. Von Struensee, S. Bierer, M. Bögemann, L. Hertle, and E. Eltze, "ExpressIon of her2/neu in locally advanced bladder cancer: implication for a molecular targeted therapy," Aktuelle Urologie, vol. 36, no. 5, pp. 423-429, 2005.

[25] M. Laé, J. Couturier, S. Oudard, F. Radvanyi, P. Beuzeboc, and A. Vieillefond, "Assessing HER2 gene amplification as a potential target for therapy in invasive urothelial bladder cancer with a standardized methodology: results in 1005 patients," Annals of Oncology, vol. 21, no. 4, pp. 815-819, 2009.

[26] J. S. Ross, J. A. Fletcher, K. J. Bloom et al., "Targeted therapy in breast cancer: the HER-2/neu gene and protein," Molecular \& Cellular Proteomics, vol. 3, no. 4, pp. 379-398, 2004.

[27] L. Albarello, L. Pecciarini, and C. Doglioni, "HER2 testing in gastric cancer," Advances in Anatomic Pathology, vol. 18, no. 1, pp. 53-59, 2011.

[28] F. De Vita, F. Giuliani, N. Silvestris, G. Catalano, F. Ciardiello, and M. Orditura, "Human epidermal growth factor receptor 2 (HER2) in gastric cancer: a new therapeutic target," Cancer Treatment Reviews, vol. 36, no. 3, pp. S11-S15, 2010.

[29] S. Krüger, G. Weitsch, H. Büttner et al., "HER2 overexpression in muscle-invasive urothelial carcinoma of the bladder: prognostic implications," International Journal of Cancer, vol. 102, no. 5, pp. 514-518, 2002.

[30] A. Alexa, F. Baderca, D. E. Zăhoi, R. Lighezan, D. Izvernariu, and M. Raica, "Clinical significance of Her2/neu overexpression in urothelial carcinomas," Romanian Journal of Morphology and Embryology, vol. 51, no. 2, pp. 277-282, 2010.

[31] P. H. Abbosh, D. J. McConkey, and E. R. Plimack, "Targeting signaling transduction pathways in bladder cancer," Current Oncology Reports, vol. 17, no. 12, article 58, 2015.

[32] H. Tsuruta, H. Kishimoto, T. Sasaki et al., "Hyperplasia and carcinomas in Pten-deficient mice and reduced PTEN protein in human bladder cancer patients," Cancer Research, vol. 66, no. 17, pp. 8389-8396, 2006.

[33] F. M. Platt, C. D. Hurst, C. F. Taylor, W. M. Gregory, P. Harnden, and M. A. Knowles, "Spectrum of phosphatidylinositol 3-kinase pathway gene alterations in bladder cancer," Clinical Cancer Research, vol. 15, no. 19, pp. 6008-6017, 2009.

[34] L. Schultz, R. Albadine, J. Hicks et al., "Expression status and prognostic significance of mammalian target of rapamycin pathway members in urothelial carcinoma of urinary bladder after cystectomy," Cancer, vol. 116, no. 23, pp. 5517-5526, 2010.

[35] C. Cordon-Cardo, "Molecular alterations associated with bladder cancer initiation and progression," Scandinavian Journal of Urology and Nephrology, vol. 42, no. 218, pp. 154-165, 2008.

[36] W. Tian and J. I. Epstein, "Invasive low-grade papillary urothelial carcinoma: an immunohistochemical study of 26 cases," Human Pathology, vol. 46, no. 12, pp. 1836-1841, 2015.

[37] M. Castillo-Martin, J. Domingo-Domenech, O. Karni-Schmidt, T. Matos, and C. Cordon-Cardo, "Molecular pathways of urothelial development and bladder tumorigenesis," Urologic Oncology: Seminars and Original Investigations, vol. 28, no. 4, pp. 401-408, 2010.

[38] N. Houédé and P. Pourquier, "Targeting the genetic alterations of the PI3K-AKT-mTOR pathway: its potential use in the treatment of bladder cancers," Pharmacology \& Therapeutics, vol. 145, pp. 1-18, 2015.

[39] I. Ahmad, J. P. Morton, L. B. Singh et al., " $\beta$-Catenin activation synergizes with PTEN loss to cause bladder cancer formation," Oncogene, vol. 30, no. 2, pp. 178-189, 2011.
[40] A. M. Puzio-Kuter, M. Castillo-Martin, C. W. Kinkade et al., "Inactivation of p53 and Pten promotes invasive bladder cancer," Genes \& Development, vol. 23, no. 6, pp. 675-680, 2009.

[41] G. Fechner, K. Claßen, D. Schmidt, S. Hauser, and S. C. Müller, "apamycin inhibits in vitro growth and release of angiogenetic factors in human bladder cancer," Urology, vol. 73, no. 3, pp. 665-668, 2009.

[42] J. J. Mansure, R. Nassim, S. Chevalier, J. Rocha, E. Scarlata, and W. Kassouf, "Inhibition of mammalian target of rapamycin as a therapeutic strategy in the management of bladder cancer," Cancer Biology and Therapy, vol. 8, no. 24, pp. 2339-2347, 2009.

[43] B. Markman, R. Dienstmann, and J. Tabernero, "Targeting the $\mathrm{PI} 3 \mathrm{~K} / \mathrm{Akt} / \mathrm{mTOR}$ pathway-beyond rapalogs," Oncotarget, vol. 1, no. 7, pp. 530-543, 2010.

[44] E. Seront, A. Pinto, C. Bouzin, L. Bertrand, J.-P. Machiels, and O. Feron, "PTEN deficiency is associated with reduced sensitivity to mTOR inhibitor in human bladder cancer through the unhampered feedback loop driving PI3K/Akt activation," British Journal of Cancer, vol. 109, no. 6, pp. 1586-1592, 2013.

[45] A. Bagrodia, L.-M. Krabbe, B. A. Gayed et al., "Evaluation of the prognostic significance of altered mammalian target of rapamycin pathway biomarkers in upper tract urothelial carcinoma," Urology, vol. 84, no. 5, pp. 1134-1140, 2014.

[46] R. T. Bryan, "Cell adhesion and urothelial bladder cancer: the role of cadherin switching and related phenomena," Philosophical Transactions of the Royal Society of London, Series B: Biological Sciences, vol. 370, no. 1661, Article ID 20140042, 2015. 


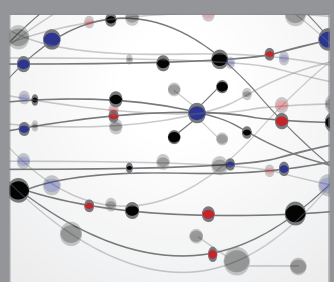

The Scientific World Journal
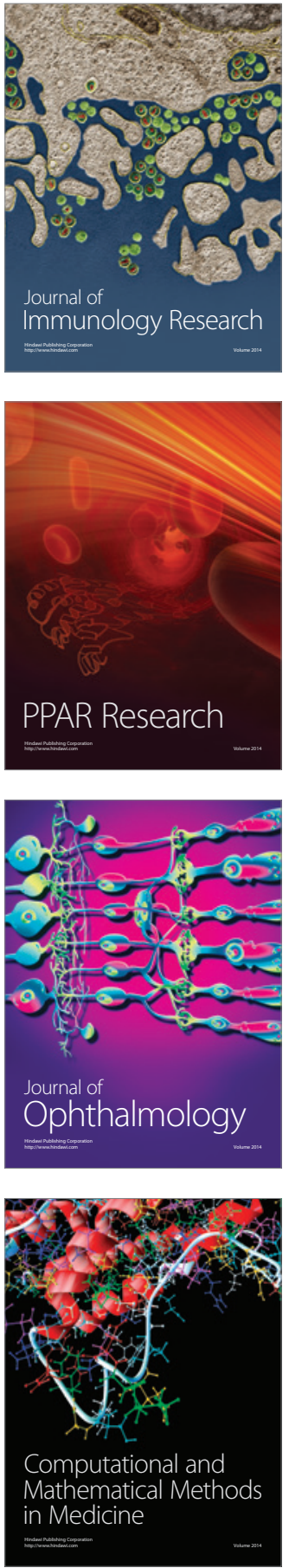

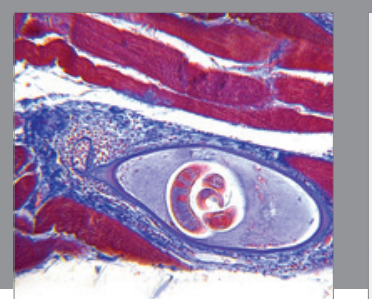

Gastroenterology Research and Practice
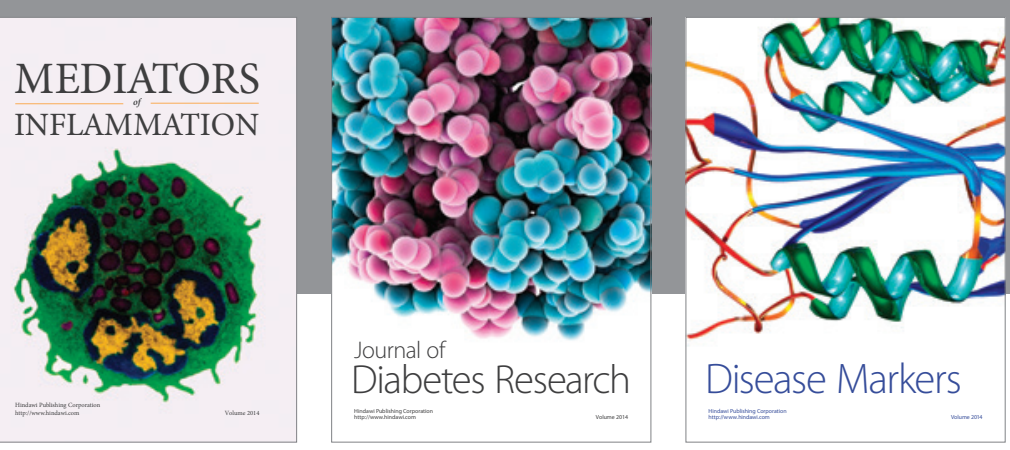

Disease Markers

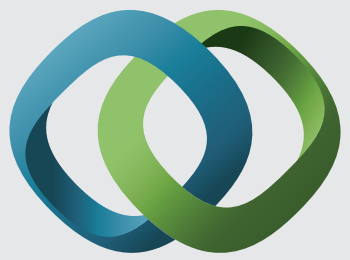

\section{Hindawi}

Submit your manuscripts at

https://www.hindawi.com
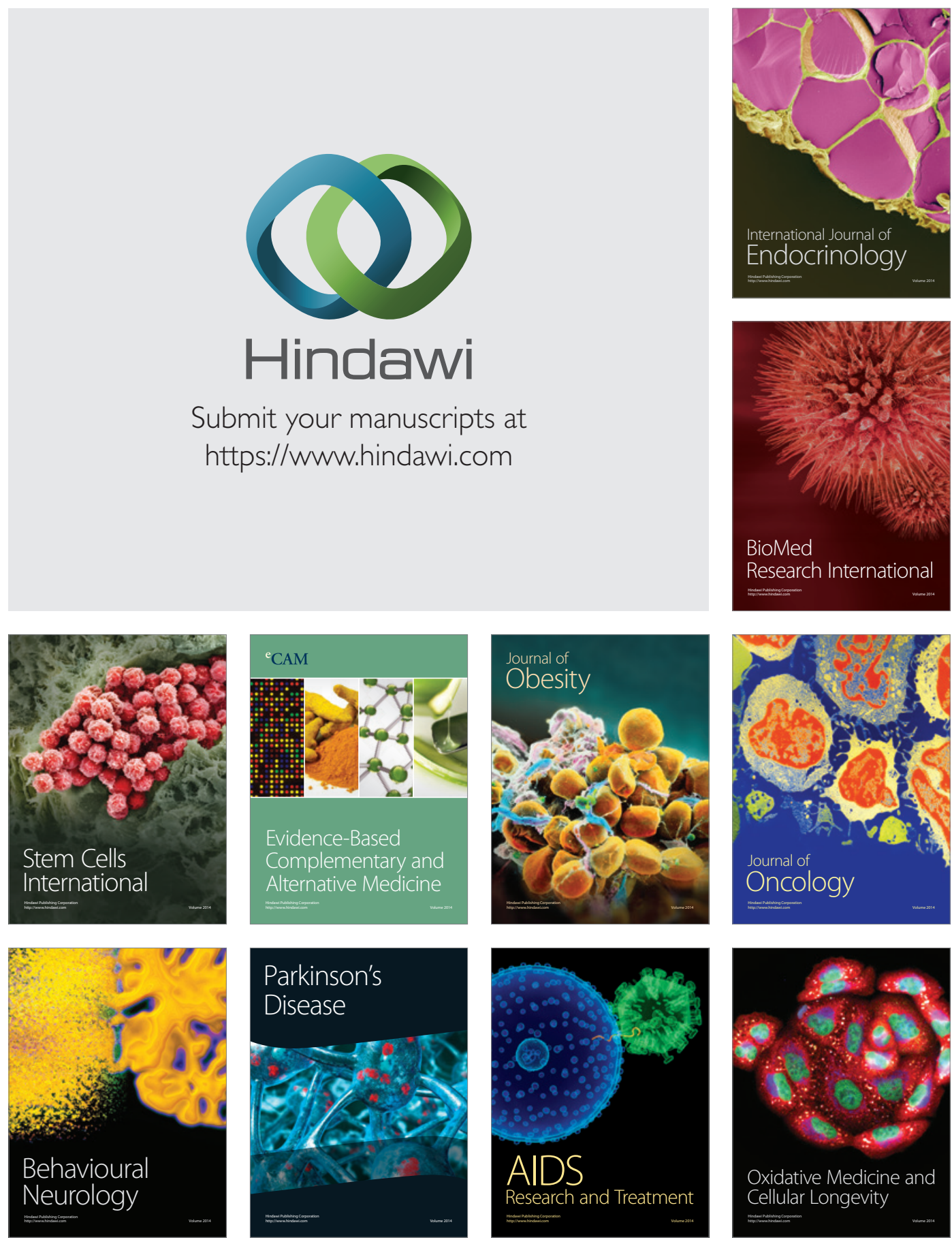